\title{
Propensity of people of African descent towards hypertension-associated cardiovascular pathologies
}

\author{
Rhena Delport \\ Hypertension Research (2016) 39, 628-630; doi:10.1038/hr.2016.69; published online 16 June 2016
}

$\mathrm{J}_{\mathrm{p}}^{\mathrm{s}=\mathrm{m}}$ ansen van Vuren et al. ${ }^{1}$ in 'Hyperpulsatile pressure, systemic inflammation and cardiac stress are associated with cardiac wall remodeling in an African male cohort: the SABPA study' explored factors associated with cardiac wall remodeling in a bi-ethnic and bi-sex cohort of school teachers in South Africa. The median ages for the ethnic groups were 44 (Caucasian) and 47 (African) years. The factors unique to African males that were associated with cardiac wall remodeling were identified. Hypertension was more prevalent in Africans than in Caucasians (54 vs. 25\%). Africans had more silent ischemic events and a higher degree of left ventricular hypertrophy. Inflammatory marker concentrations were significantly higher in the African cohort. Within the male cohort, Africans, following correction for covariates had significantly more silent ischemic events, a higher degree of left ventricular hypertrophy, higher blood pressure measurements and more inflammatory markers than Caucasians. Similar differences were observed for the female cohort, with the exception of the number of ischemic events that did not differ significantly. The authors conclude that cardiac wall remodeling in African males is associated with hyperpulsatile pressure, low-grade systemic inflammation and indices of ventricular stress.

The mechanism of cardiac remodeling in black Africans is explained as follows:

- Tumor necrosis factor (TNF)- $\alpha$ release induces apoptosis, promotes release of reactive oxygen species and reduces nitric oxide generation. Increase in myocardial

$\mathrm{R}$ Delport is at Department of Chemical Pathology, School of Medicine, Faculty of Health Sciences, University of Pretoria, Pretoria, South Africa

E-mail: rhena.delport@up.ac.za afterload results from the impairment of endothelium-dependent vasodilation, as well as from the concomitant excessive $\alpha$-adrenergic responsiveness, triggering cardiac remodeling.

- Interleukin-6 gives rise to increased tissue factor and von Willebrand factor release, thereby increasing collagen deposition.

- Arterial stiffness increases owing to increased vascular wall permeability leading to an increase in ventricular transmural pressure and volume overload, which induces stretching of the myocytes and results in cardiac remodeling.

- Inactivity, smoking and alcohol use compound the insult of hypertension.

The novelty of this small study lies in its composite nature, where biochemical markers and cardiac indices represent ventricular challenges associated with hypertension. Ethnicity may be an important factor in predicting hypertension, which may evolve into cardiac failure or ischemic heart disease. ${ }^{2}$ Findings linking ethnicity, hypertension, pro-inflammatory status or ventricular remodeling in systolic hypertension are not novel. What is interesting is that the higher frequency of silent ischemic events in African males was not associated with higher than expected (for black race or for degree of ventricular strain) Troponin $\mathrm{T}$ (Trop $\mathrm{T}$ ) and NT-proBNP concentrations. The significant relationship of NT-proBNP with TNF- $\alpha$, Trop $\mathrm{T}$ and pulse pressure supports the construct that ventricular remodeling is related to both inflammation and myocardial ischemia in the presence of hypertension, as an African-specific phenomenon.

This commentary reflects on the magnitude of the rise in incidence of hypertension specifically in Africa, and the propensity of people of African descent toward hypertension-associated cardiovascular pathologies. Some additional comments are made on the diagnostic and prognostic usefulness of cardiac markers in hypertension-related cardiovascular disease resulting in morbidity and mortality.

\section{RISING INCIDENCE OF HYPERTENSION IN AFRICA}

Concern about the rapid increase in cardiovascular disease risk factors and hypertension, ultimately resulting in a higher prevalence of atherosclerosis and ischemic heart disease, in Sub-Saharan Africa has been raised. Deaths attributed to high systolic blood pressure have been estimated at 10.4 million for 1990-2013, and disability-adjusted life-years have been estimated at 208.1 million in the Global Burden of Disease Study 2013. ${ }^{3}$ A high prevalence of hypertension and low percentages of hypertension awareness, treatment and control were reported in a systematic review and meta-analysis assessing the burden of hypertension in Sub-Saharan Africa. ${ }^{4}$ Studies published between 2000 and 2013 were included in the analysis, and identified 110414 participants. Prevalence of hypertension was predicted at mean participant ages of $30,40,50$ and 60 years, representing 16, 26, 35 and $44 \%$, respectively, and the overall prevalence was 30\% (95\% confidence interval (CI), 27-34\%). ${ }^{4}$ The relevance of this study, ${ }^{1}$ performed in participants with similar socioeconomic status, is substantiated by the realization of covert risk factor and cardiovascular dysfunction prevalent in South Africans, and there are potential implications related to the burden of cardiovascular disease in the continent of Africa. 


\section{HYPERTENSION-ASSOCIATED CARDIOVASCULAR PATHOLOGIES IN AFRICANS}

The Bogalusa Heart Study found that blood pressure variability, diet, salt intake and other environmental effects had a greater damaging effect on the hearts of black Americans compared with Caucasian Americans owing to differing autonomic and metabolic responses to stress. ${ }^{2}$ Support for ethnically inked cardiometabolic differences is found in this South African cohort, which associates vascular responsiveness with ventricular distress expressed as hypertensive left ventricular structural changes and the number of silent ischemic events. ${ }^{5}$ Although many abnormalities associated with hypertensive left ventricular hypertrophy are common to heart failure with preserved ejection fraction, ${ }^{6}$ hypertension as a cause of heart failure reportedly increased from 23 to $45 \%$ in the sub-Saharan Africa Survey of Heart Failure, whereas ischemic heart disease as a cause of heart failure increased from 2 to nearly $8 \%{ }^{7}$

\section{DIAGNOSTIC AND PROGNOSTIC USEFULNESS OF CARDIAC MARKERS IN HYPERTENSION-RELATED CARDIOVASCULAR DISEASE}

Contrary to expectation, African participants had lower Trop $\mathrm{T}$ concentrations than Caucasian participants. Notably, the meanadjusted values for all the four groups, differentiated by gender and ethnicity, were well above the detection limit of the assay $\left(3 \mathrm{pg} \mathrm{ml}^{-1}\right)$. The 75 th percentile value was $6.30 \mathrm{pg} \mathrm{ml}^{-1}$ for African participants and $8.63 \mathrm{pg} \mathrm{ml}^{-1}$ for Caucasian participants, whereas the 99th percentile cut point for the diagnosis of myocardial infarction is $14 \mathrm{ng} \mathrm{ml}^{-1}$. Only $26.2 \%$ of the total study population had undetectable Trop $\mathrm{T}$ values. A general population study (Dallas County) found detectable Trop $\mathrm{T}$ concentrations in $34.4 \%$ (95\% CI, 30.6-38.3\%) of black subjects and $25.4 \%$ (95\% CI, 21.2-29.0\%) of Caucasian subjects in participants aged 40-50 years. ${ }^{8}$ The authors conclude that Trop $\mathrm{T}$, detected with a high-sensitivity method, 'was associated with structural heart disease and subsequent risk for all-cause mortality'. ${ }^{8}$ The contrast with the Jansen van Vuren ${ }^{1}$ study exemplifies the possible extent of cardiovascular disease risk for both the South African ethnic groups. Furthermore, risk for the progression of left ventricular hypertrophy to heart failure and cardiovascular death is implicated by Trop T and NT-proBNP levels above the age- and sex-specific 75th percentile of the population. ${ }^{9}$ Both highsensitivity Trop T and NT-proBNP are valid predictors of adverse events in the general population. ${ }^{10}$ It is advisable that serial measurements of these biomarkers, among other indicators with adequate sensitivity, are employed for timely diagnosis and appropriate management of ventricular remodeling, independent of the presence of myocardial ischemia, when increases in blood pressure are detected in the general population.

\section{CONFLICT OF INTEREST}

The author declares no conflict of interest.

1 Jansen van Vuren E, Malan L, von Känel R, Cockeran M, Malan NT. Hyperpulsatile pressure, systemic inflammation and cardiac stress are associated with cardiac wall remodelling in an African male cohort: the SABPA study. Hypertens Res 2016; 39: 648-653.

2 Berenson GS, Chen W, Dasmahapatra P, Fernandez C, Giles T, Xu J, Srinivasan SR. Stimulus response of blood pressure in black and white young individuals helps explain racial divergence in adult cardiovascular disease: the Bogalusa Heart Study. J Am Soc Hypertens 2011; 5: 230-238.

3 GBD 2013 Risk Factors Collaborators, Forouzanfar MH, Alexander L, Anderson HR, Bachman VF, Biryukov S, Brauer M, Burnett R, Casey D, Coates MM, Cohen A, Delwiche K, Estep K, Frostad JJ, Astha KC, Kyu HH, Moradi-Lakeh M, Ng M, Slepak EL, Thomas BA, Wagner J, Aasvang GM, Abbafati C, Abbasoglu Ozgoren A, Abd-Allah F, Abera SF, Aboyans V, Abraham B, Abraham JP, Abubakar I, Abu-Rmeileh NM, Aburto TC, Achoki T, Adelekan A, Adofo K, Adou AK, Adsuar JC, Afshin A, Agardh EE, Al Khabouri MJ, Al Lami FH, Alam SS, Alasfoor D, Albittar MI, Alegretti MA, Aleman AV, Alemu ZA, Alfonso-Cristancho R, Alhabib S, Ali R, Ali MK, Alla F, Allebeck P, Allen PJ, Alsharif U, Alvarez E, Alvis-Guzman N, Amankwaa AA, Amare AT, Ameh EA, Ameli O, Amini H, Ammar W, Anderson BO, Antonio CA, Anwari P, Argeseanu Cunningham S, Arnlöv J, Arsenijevic VS, Artaman A, Asghar RJ, Assadi R, Atkins LS, Atkinson C, Avila MA, Awuah B, Badawi A, Bahit MC, Bakfalouni T, Balakrishnan K, Balalla S, Balu RK, Banerjee A, Barber RM, Barker-Collo SL, Barquera S, Barregard L, Barrero LH, Barrientos-Gutierrez T, Basto-Abreu AC, Basu A, Basu S, Basulaiman MO, Batis Ruvalcaba C, Beardsley J, Bedi N, Bekele T, Bell ML, Benjet C, Bennett DA, Benzian $H$, Bernabé E, Beyene TJ, Bhala N, Bhalla A, Bhutta ZA, Bikbov B, Bin Abdulhak AA, Blore JD, Blyth FM, Bohensky MA, Bora Başara B, Borges G, Bornstein NM, Bose D, Boufous S, Bourne RR, Brainin M, Brazinova A, Breitborde $N J$, Brenner $H$, Briggs $A D$, Broday DM, Brooks PM, Bruce NG, Brugha TS, Brunekreef B, Buchbinder R, Bui LN, Bukhman G, Bulloch AG, Burch M, Burney PG, Campos-Nonato IR, Campuzano JC, Cantoral AJ, Caravanos J, Cárdenas R, Cardis E, Carpenter DO Caso V, Castañeda-Orjuela CA, Castro RE, Catalá-López F, Cavalleri F, Çavlin A, Chadha VK, Chang JC, Charlson FJ, Chen H, Chen W, Chen Z, Chiang PP, Chimed-Ochir O, Chowdhury R, Christophi CA, Chuang TW, Chugh SS, Cirillo M, Claßen TK, Colistro V, Colomar M, Colquhoun SM, Contreras AG, Cooper C, Cooperrider K, Cooper LT, Coresh J, Courville KJ, Criqui MH, Cuevas-Nasu L, Damsere-Derry J, Danawi H, Dandona L, Dandona R, Dargan PI, Davis A, Davitoiu DV, Dayama A, de Castro EF, De la Cruz-Góngora V, De Leo D, de Lima G, Degenhardt L, del Pozo-Cruz B, Dellavalle RP, Deribe K, Derrett S, Des Jarlais DC, Dessalegn M, de Veber GA, Devries KM, Dharmaratne $\mathrm{SD}$, Dherani MK, Dicker D, Ding EL, Dokova K, Dorsey ER, Driscoll TR, Duan L, Durrani AM,
Ebel BE, Ellenbogen RG, Elshrek YM, Endres M, Ermakov SP, Erskine HE, Eshrati B, Esteghamati A, Fahimi S, Faraon EJ, Farzadfar F, Fay DF, Feigin VL, Feigl AB, Fereshtehnejad SM, Ferrari AJ, Ferri CP, Flaxman AD, Fleming TD, Foigt $\mathrm{N}$, Foreman $\mathrm{KJ}$, Paleo UF, Franklin RC, Gabbe B, Gaffikin L, Gakidou E, Gamkrelidze A, Gankpé FG, Gansevoort RT, García-Guerra FA, Gasana E, Geleijnse JM, Gessner BD, Gething P, Gibney KB, Gillum RF, Ginawi IA, Giroud M, Giussani G, Goenka S, Goginashvili K, Gomez Dantes H, Gona P, Gonzalez de Cosio T, González-Castell D, Gotay CC, Goto A, Gouda HN Guerrant RL, Gugnani HC, Guillemin F, Gunnell D, Gupta R, Gupta R, Gutiérrez RA, Hafezi-Nejad N, Hagan $H$, Hagstromer M, Halasa YA, Hamadeh RR, Hammami M, Hankey GJ, Hao Y, Harb HL, Haregu TN, Haro JM, Havmoeller R, Hay SI, Hedayati MT, Heredia-Pi IB, Hernandez L, Heuton KR, Heydarpour P, Hijar M, Hoek HW, Hoffman HJ, Hornberger JC, Hosgood HD, Hoy DG, Hsairi M, $\mathrm{Hu}$ G, Hu H, Huang C, Huang JJ, Hubbell BJ, Huiart L, Husseini A, lannarone ML, Iburg KM, Idrisov $\mathrm{BT}$, Ikeda $\mathrm{N}$, Innos $\mathrm{K}$, Inoue $\mathrm{M}$, Islami $\mathrm{F}$, Ismayilova $S$, Jacobsen $K H$, Jansen $H A$, Jarvis DL, Jassal SK, Jauregui A, Jayaraman S, Jeemon $P$, Jensen $P N$, Jha $V$, Jiang $F$, Jiang $G$, Jiang $Y$, Jonas JB, Juel K, Kan $H$, Kany Roseline SS, Karam NE, Karch A, Karema CK, Karthikeyan G, Kaul A, Kawakami N, Kazi DS, Kemp AH, Kengne AP, Keren A, Khader YS, Khalifa SE, Khan EA, Khang YH, Khatibzadeh S, Khonelidze I, Kieling C, Kim D, Kim S, Kim Y, Kimokoti RW, Kinfu $Y$, Kinge JM, Kissela BM, Kivipelto $M$, Knibbs LD, Knudsen AK, Kokubo Y, Kose MR, Kosen S, Kraemer A, Kravchenko M, Krishnaswami S, Kromhout H, Ku T, Kuate Defo B, Kucuk Bicer B, Kuipers EJ, Kulkarni C, Kulkarni VS, Kumar GA, Kwan GF, Lai T, Lakshmana Balaji A, Lalloo R, Lallukka T, Lam H, Lan Q, Lansingh VC, Larson HJ, Larsson A, Laryea DO, Lavados PM, Lawrynowicz AE, Leasher JL, Lee JT, Leigh J, Leung R, Levi M, Li Y, Li Y, Liang J, Liang X, Lim SS, Lindsay MP, Lipshultz SE, Liu S, Liu Y, Lloyd BK, Logroscino G, London SJ, Lopez N Lortet-Tieulent J, Lotufo PA, Lozano R, Lunevicius R, Ma J, Ma S, Machado VM, Maclntyre MF, Magis-Rodriguez C, Mahdi AA, Majdan M, Malekzadeh R, Mangalam S, Mapoma CC, Marape M, Marcenes W, Margolis DJ, Margono C, Marks GB, Martin RV, Marzan MB, Mashal MT, Masiye F, Mason-Jones AJ, Matsushita K, Matzopoulos R, Mayosi BM, Mazorodze TT, McKay AC, McKee M, McLain A, Meaney PA, Medina C, Mehndiratta MM, Mejia-Rodriguez F, Mekonnen W, Melaku YA, Meltzer M, Memish ZA, Mendoza W, Mensah GA, Meretoja A, Mhimbira FA, Micha R, Miller TR, Mills EJ, Misganaw A, Mishra S, Mohamed Ibrahim N, Mohammad KA, Mokdad AH, Mola GL, Monasta L, Montañez Hernandez JC, Montico M, Moore AR, Morawska L, Mori R, Moschandreas J, Moturi WN, Mozaffarian D, Mueller UO, Mukaigawara M Mullany EC, Murthy KS, Naghavi M, Nahas Z, Naheed A, Naidoo KS, Naldi L, Nand D, Nangia V, Narayan KM, Nash D, Neal B, Nejjari C, Neupane SP, Newton CR, Ngalesoni FN, Ngirabega Jde D, Nguyen G, Nguyen NT, Nieuwenhuijsen MJ, Nisar MI, Nogueira JR, Nolla JM, Nolte S, Norheim OF, Norman RE, Norrving B, Nyakarahuka L, Oh IH, Ohkubo T, Olusanya BO, Omer SB, Opio JN, Orozco R, Pagcatipunan RS Jr, Pain AW, Pandian JD, Panelo $\mathrm{Cl}$, Papachristou C, Park EK, Parry CD, Paternina Caicedo AJ, Patten SB, Paul VK, Pavlin BI, Pearce N, Pedraza LS, Pedroza A, Pejin Stokic L, Pekericli A, Pereira DM, Perez-Padilla R, Perez-Ruiz F, Perico N, Perry SA, Pervaiz A, Pesudovs K, Peterson CB, Petzold M, Phillips MR, Phua HP, Plass D, Poenaru D, Polanczyk GV, Polinder S, Pond CD, Pope CA, Pope D, Popova S, Pourmalek F, Powles J, Prabhakaran D, Prasad NM, Qato DM, Quezada AD, Quistberg DA, Racapé L, Rafay A, Rahimi K, Rahimi-Movaghar V, Rahman SU, Raju M, Rakovac I, Rana SM, Rao M, Razavi H, Reddy KS, 
Refaat AH, Rehm J, Remuzzi G, Ribeiro AL, Riccio PM, Richardson L, Riederer A, Robinson M Roca A, Rodriguez A, Rojas-Rueda D, Romieu I, Ronfani L, Room R, Roy N, Ruhago GM, Rushton L, Sabin N, Sacco RL, Saha S, Sahathevan R, Sahraian MA, Salomon JA, Salvo D, Sampson UK, Sanabria JR, Sanchez LM, Sánchez-Pimienta TG, Sanchez-Riera L, Sandar L, Santos IS, Sapkota A Satpathy M, Saunders JE, Sawhney M, Saylan MI, Scarborough $P$, Schmidt JC, Schneider IJ, Schöttker B, Schwebel DC, Scott JG, Seedat S, Sepanlou SG, Serdar B, Servan-Mori EE, Shaddick G, Shahraz S, Levy TS, Shangguan S, She J, Sheikhbahaei S, Shibuya K, Shin $\mathrm{HH}$ Shinohara Y, Shiri R, Shishani K, Shiue I, Sigfusdottir ID, Silberberg DH, Simard EP, Sindi S, Singh A, Singh GM, Singh JA, Skirbekk V, Sliwa K, Soljak M, Soneji S, Søreide K, Soshnikov S, Sposato LA, Sreeramareddy CT, Stapelberg NJ, Stathopoulou V, Steckling N, Stein DJ, Stein MB, Stephens N, Stöckl H, Straif K, Stroumpoulis K Sturua L, Sunguya BF, Swaminathan S, Swaroop M Sykes BL, Tabb KM, Takahashi K, Talongwa RT, Tandon N, Tanne D, Tanner M, Tavakkoli M, Te Ao BJ, Teixeira CM, Téllez Rojo MM, Terkawi AS, Texcalac-Sangrador JL, Thackway SV, Thomson B, Thorne-Lyman AL, Thrift AG, Thurston GD, Tillmann T, Tobollik M, Tonelli M, Topouzis F, Towbin JA, Toyoshima H, Traebert J, Tran BX, Trasande L, Trillini M, Trujillo U, Dimbuene ZT, Tsilimbaris $M$, Tuzcu EM, Uchendu US, Ukwaja KN, Uzun SB, van de Vijver S, Van Dingenen R, van Gool CH, van Os J, Varakin YY, Vasankari TJ, Vasconcelos AM, Vavilala
MS, Veerman LJ, Velasquez-Melendez G, Venketasubramanian N, Vijayakumar L, Villalpando S, Violante FS, Vlassov VV, Vollset SE, Wagner GR, Waller SG, Wallin MT, Wan X, Wang H, Wang J, Wang L, Wang W, Wang $\mathrm{Y}$, Warouw TS, Watts $\mathrm{CH}$, Weichenthal $\mathrm{S}$, Weiderpass $E$, Weintraub RG, Werdecker A, Wessells KR, Westerman R, Whiteford HA, Wilkinson JD, Williams HC, Williams TN, Woldeyohannes SM, Wolfe CD, Wong JQ, Woolf AD, Wright JL, Wurtz B, Xu G, Yan LL, Yang G, Yano $Y$, Ye $P$, Yenesew $M$, Yentür GK, Yip P, Yonemoto N, Yoon SJ, Younis MZ, Younoussi Z, Yu C, Zaki ME, Zhao Y, Zheng Y, Zhou M, Zhu J, Zhu S, Zou X, Zunt JR, Lopez AD, Vos T, Murray CJ. Global, regional, and national comparative risk assessment of 79 behavioural, environmental and occupational, and metabolic risks or clusters of risks in 188 countries, 1990-2013: a systematic analysis for the Global Burden of Disease Study 2013. Lancet 2015; 386: 2287-2323.

4 Ataklte F, Erqou S, Kaptoge S, Taye B, EchouffoTcheugui JB, Kengne AP. Burden of undiagnosed hypertension in sub-saharan Africa: a systematic review and meta-analysis. Hypertension 2015; 65: 291-298.

5 Malan L, Hamer M, Schlaich MP, Lambert GW, Harvey BH, Reimann M, Lambert GW, Harvey BH, Reimann M, Ziemssen $T$, de Geus EJ, Huisman HW, van Rooyen JM, Schutte R, Schutte AE, Fourie CM, Seedat YK, Malan NT. Facilitated defensive coping, silent ischemia and ECG left-ventricular hypertrophy: the SABPA study. J Hypertens 2012; 30: 543-550.

6 Paulus WJ, Tschöpe C. A novel paradigm for heart failure with preserved ejection fraction: comorbidities drive myocardial dysfunction and remodeling through coronary microvascular endothelial inflammation. J Am Coll Cardiol 2013; 62 : 263-271.

7 Damasceno A, Mayosi BM, Sani M, Ogah OS, Mondo C, Ojji D, Dzudie A, Kouam CK, Suliman A, Schrueder N, Yonga G, Ba SA, Maru F, Alemayehu B, Edwards C, Davison BA, Cotter G, Sliwa K. The causes, treatment, and outcome of acute heart failure in 1006 Africans from 9 countries: results of the sub-Saharan Africa Survey of Heart Failure. Arch Int Med 2012; 172: 1386-1394.

8 de Lemos JA, Drazner $\mathrm{MH}$, Omland $\mathrm{T}$, Ayers $\mathrm{CR}$, Khera A, Rohatgi A, Hashim I, Berry JD, Das SR, Morrow DA, McGuire DK. Association of troponin $T$ detected with a highly sensitive assay and cardiac structure and mortality risk in the general population. JAMA 2010; 304: 2503-2512.

9 Neeland IJ, Drazner MH, Berry JD, Ayers CR, deFilippi C, Seliger SL, Hashim I, Berry JD, Das SR, Morrow DA, McGuire DK. Biomarkers of chronic cardiac injury and hemodynamic stress identify a malignant phenotype of left ventricular hypertrophy in the general population. J Am Coll Cardiol 2013; 61: 187-195.

10 Nambi V, Liu X, Chambless LE, de Lemos JA, Virani SS, Agarwal S, Boerwinkle E, Hoogeveen RC, Aguilar D, Astor BC, Srinivas PR, Deswal A, Mosley TH, Coresh J, Folsom AR, Heiss G, Ballantyne CM. Troponin T and $\mathrm{N}$-terminal pro-B-type natriuretic peptide: a biomarker approach to predict heart failure risk-the atherosclerosis risk in communities study. Clin Chem 2013; 59: 1802-1810. 